26:2813-2820

7. Barrallo-Gimeno, A., and Nieto, M.A. 2005. The Snail genes as inducers of cell movement and survival: implications in development and cancer. Development. 132:3151-3161.

8. Fuchs, E., Tumbar, T., and Guasch, G. 2004. Socializing with the neighbors: stem cells and their niche. Cell. 116:769-778.

9. Lapidot, T., et al. 1994. A cell initiating human acute myeloid leukaemia after transplantation into SCID mice. Nature. 367:645-648.

10. Al-Haji, M., Wicha, M.S., Benito-Hernandez, A., Morrison, S.J., and Clarke, M.F. 2003. Prospective identification of tumorigenic breast cancer cells. Proc. Natl. Acad. Sci. U. S. A. 100:3983-3988.

11. O'Brien, C.A., Pollett, A., Gallinger, S., and Dick, J.E. 2007. A human colon cancer cell capable of initiating tumour growth in immunodeficient mice.
Nature. 445:106-110.

12. Hermann, P.C., et al. 2007. Distinct populations of cancer stem cells determine tumor growth and metastatic activity in human pancreatic cancer. Cell Stem Cell. 1:313-323.

13. Ricci-Vitiani, L., et al. 2007. Identification and expansion of human colon-cancer-initiating cells. Nature. 445:111-115.

14. Singh, S.K., et al. 2004. Identification of human brain tumour initiating cells. Nature. 432:396-401.

15. McCoy, E.L., et al. 2009. Six1 expands the mouse mammary epithelial stem/progenitor cell pool and induces mammary tumors that undergo epithelial-mesenchymal transition. J. Clin. Invest. 119:2663-2677.

16. Micalizzi, D.S., et al. 2009. The Six1 homeoprotein induces human mammary carcinoma cells to undergo epithelial-mesenchymal transition and metastasis in mice through increasing TGF- $\beta$ signaling. J. Clin. Invest. 119:2678-2690.

17. Abate-Shen, C. 2002. Deregulated homeobox gene expression in cancer: cause or consequence? Nat. Rev. Cancer. 2:777-785.

18. Ford, H.L., Kabingu, E.N., Bump, E.A., Mutter, G.L., and Pardee, A.B. 1998. Abrogation of the G2 cell cycle checkpoint associated with overexpression of HSIX1: a possible mechanism of breast carcinogenesis. Proc. Natl. Acad. Sci. U. S. A. 95:12608-12613.

19. Reichenberger, K.J., Coletta, R.D., Schulte, A.P., Varella-Garcia, M., and Ford, H.L. 2005. Gene amplification is a mechanism of Six 1 overexpression in breast cancer. Cancer Res. 65:2668-2675.

20. Coletta, R.D., et al. 2008. Six1 overexpression in mammary cells induces genomic instability and is sufficient for malignant transformation. Cancer Res. 68:2204-2213.

\title{
Endocytic control of ion channel density as a target for cardiovascular disease
}

\section{Gail A. Robertson}

Department of Physiology, University of Wisconsin — Madison, Madison, Wisconsin, USA.

\begin{abstract}
Ion channels encoded by the human ether-a-go-go-related gene (HERG) give rise to the rapidly activating delayed rectifier $\mathrm{K}^{+}$current $\left(\mathrm{I}_{\mathrm{Kr}}\right)$, the perturbation of which causes ventricular arrhythmias associated with inherited and acquired long QT syndrome. Electrolyte imbalances, such as reduced serum $\mathrm{K}^{+}$levels (hypokalemia), also trigger these potentially fatal arrhythmias. In this issue of the JCI, Guo et al. report that physiological levels of serum $\mathrm{K}^{+}$ are required to maintain normal HERG surface density in HEK 293 cells and $I_{K r}$ in rabbit cardiomyocytes. They found that hypokalemia evoked HERG channel ubiquitination, enhanced internalization via endocytosis, and ultimately degradation at the lysosome, thus identifying unbridled turnover as a mechanism of hypokalemia-induced arrhythmia. But too little channel turnover can also cause disease, as suggested by Kruse et al. in a study also in this issue. The authors identified mutations in TRPM4 - a nonselective cation channel - in a large family with progressive familial heart block type I and showed that these mutations prevented channel internalization (see the related articles beginning on pages 2745 and 2737, respectively).
\end{abstract}

How can a drop in serum $\mathrm{K}^{+}$levels cause sudden cardiac death? Hypokalemia has long been recognized as a risk factor for potentially catastrophic Torsades de pointes $(\mathrm{TdP})$ ventricular arrhythmias $(1,2)$. TdP is often associated with long QT syndrome, characterized by delayed repolarization after excitation of the heart and a corresponding increase of the QT interval on the ECG (Figure 1). Delayed repolarization arises when ventricular action potentials are prolonged, leading to electrical instabilities at both the cellular and the tissue level that

Conflict of interest: The author has declared that no conflict of interest exists.

Citation for this article: J. Clin. Invest. 119:2531-2534 (2009). doi:10.1172/JCI40427. trigger arrhythmia. A critical determinant of action potential duration (APD) is the so-called rapidly activating delayed rectifier $\mathrm{K}^{+}$channel current $\left(\mathrm{I}_{\mathrm{Kr}}\right.$; ref. 3$)$. Sanguinetti and colleagues demonstrated that $\mathrm{I}_{\mathrm{Kr}}$ block causes APD prolongation in ventricular myocytes (3), and $\mathrm{I}_{\mathrm{Kr}}$ amplitude paradoxically diminishes in low levels of extracellular $\mathrm{K}^{+}$concentration $\left(\left[\mathrm{K}^{+}\right]_{\mathrm{o}}\right)$ despite increased concentration gradient and driving force (4). Thus, $\mathrm{I}_{\mathrm{Kr}}$ emerged as a potential target for hypokalemia-induced TdP.

When it was subsequently determined that $\mathrm{I}_{\mathrm{Kr}}$ was produced by assemblies of subunits encoded by the human ether-a-go-gorelated gene (HERG; refs. 5, 6), analysis of gating mechanisms in the heterologously expressed HERG channel led to the first proposals for a mechanism of hypokalemiainduced reduction in $\mathrm{I}_{\mathrm{Kr}}$. The early studies showed that HERG channels respond to a depolarizing voltage command by entering a stable, inactivated state that suppresses the current; upon repolarization, inactivation recovers to unleash a large resurgent tail current (Figure 2A and refs. 5, 6). During a ventricular action potential, these gating transitions work together to ensure that the resurgent current peaks late and contributes to phase 3 repolarization (Figure $2 \mathrm{~B}$ and ref. 7 ). The suppression at positive voltages occurs by a C-type inactivation mechanism in which the conducting path collapses, a process enhanced in low $\left[\mathrm{K}^{+}\right.$。 $(8,9)$. Such a mechanism could explain a reduction in $\mathrm{I}_{\mathrm{Kr}}$ amplitude in conditions of low $\left[\mathrm{K}^{+}\right]_{\mathrm{o}}$ and was thus proposed to mediate hypokalemia-induced arrhythmia (10).

But the issue is not yet settled. The records of HERG currents in response to voltage step changes (e.g., Figure 2A) at room temperature do not readily predict the effects of hypokalemia on the native channel during an action potential at physiological temperatures. The current profile depends on multiple gating processes with distinct voltage, time, and temperature dependencies (5, $6,11)$. Reducing $\left[\mathrm{K}^{+}\right]_{\mathrm{o}}$ will enhance channel inactivation and thus further suppress current at the peak of the action potential, but the increase in driving force will have the opposite effect on current amplitude as the membrane potential begins to repolarize 


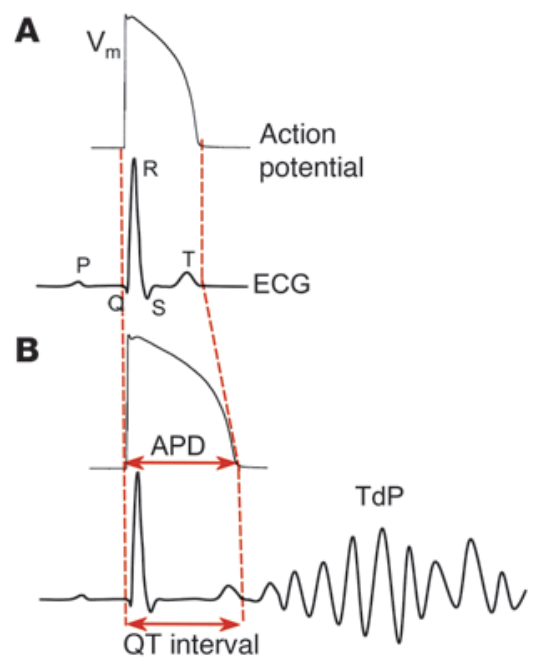

and the current rebounds from inactivation. Kinetic models could be used to predict whether the effects on gating and driving force will increase APD or facilitate TdP arrhythmias (12). Even simpler would be to measure HERG currents at physiological temperatures in response to a voltage clamp command mimicking a ventricular action potential (e.g., Figure 2B), which could reveal whether the overall current amplitudes or kinetic profiles are altered as a consequence of the complex effects of low $\left[\mathrm{K}^{+}\right]_{\mathrm{o}}$ on gating and driving force $(7,12)$.

\section{Hypokalemia accelerates HERG- specific channel turnover}

Now Guo and colleagues suggest a different mechanism for hypokalemia-induced $\mathrm{I}_{\mathrm{Kr}}$ reduction in this issue of the JCI (13). This tour de force study in heterologous and native systems examined the effects of low $\left[\mathrm{K}^{+}\right]_{\text {o }}$ on HERG current amplitudes, subunit surface expression, and trafficking, with a quantitative assessment of the concentration dependence and temporal characteristics of each $\mathrm{K}^{+}$-sensitive change. The study links these cellular mechanisms to the corresponding outcomes in vivo by evaluating the effects of a low- $\left[\mathrm{K}^{+}\right]_{\mathrm{o}}$ diet on

\section{Figure 1}

Long QT syndrome and TdP cardiac arrhythmia. (A) Action potential (upper trace) and the corresponding surface ECG (lower trace) of a normal heartbeat. The P wave, QRS complex, and $T$ wave are shown. $V_{m}$, membrane voltage. (B) APD is prolonged when $I_{\mathrm{Kr}}$ is reduced by mutation of the HERG gene or via drug block (upper trace). The QT interval of the ECG is correspondingly prolonged, and can lead to oscillating TdP arrhythmias (lower trace).
QT interval in intact rabbits and on APD and $\mathrm{I}_{\mathrm{Kr}}$ amplitude in isolated rabbit myocytes. The authors found that low $\left[\mathrm{K}^{+}\right]_{\mathrm{o}}$ causes a chronic reduction of $\mathrm{I}_{\mathrm{Kr}}$ due to clathrin-independent internalization of HERG channels and a corresponding prolongation of APD and the QT interval. The channels are first shuttled to early endosomes, which fuse to multivesicular bodies, and ultimately deposited for disposal in the lysosome (Figure 3A).

Whereas HERG currents in HEK 293 cells incubated over a period of 12 hours at nor$\mathrm{mal}\left[\mathrm{K}^{+}\right]_{\mathrm{o}}$ were stable, at $0 \mathrm{mM}\left[\mathrm{K}^{+}\right]_{\mathrm{o}}$, the currents were nearly eliminated (Figure $3 \mathrm{~A}$ and ref. 13). (Cells were placed in physiological $\left[\mathrm{K}^{+}\right]_{\mathrm{o}}$ during recording.) A corresponding loss in the mature surface channel protein was observed on Western blots of cell lysates and the biotin-purified surface population. The rate of channel internalization, measured in the presence of brefeldin A to block the insertion of newly synthesized protein, was accelerated 4-fold in $0 \mathrm{mM}\left[\mathrm{K}^{+}\right]_{\mathrm{o}}$. HERG channel proteins were found to be ubiquitinated prior to internalization, and increases in ubiquitin accelerated internalization (Figure 3A). No changes in channel biophysical properties were observed, nor did the coexpression of KCNE1 or KCNE2, potential HERG-associated subunits (14, 15), alter the channel properties or their response to incubation in low $\left[\mathrm{K}^{+}\right]_{\mathrm{o}}$.

Of course, $0 \mathrm{mM}\left[\mathrm{K}^{+}\right]_{\mathrm{o}}$ is not the same as hypokalemia, which is clinically defined as a serum $\left[\mathrm{K}^{+}\right]_{\mathrm{o}}$ level below $3.5 \mathrm{mM}$ and has been previously reported in humans at levels as low as $1.2 \mathrm{mM}(16,17)$. To evaluate the effects of reduced $\left[\mathrm{K}^{+}\right]_{\mathrm{o}}$ on $\mathrm{I}_{\mathrm{Kr}}$ in vivo, rabbits were made hypokalemic by a low- $\mathrm{K}^{+}$diet (13). At an average serum $\left[\mathrm{K}^{+}\right]_{\mathrm{o}}$ of $2.4 \mathrm{mM}$,

\section{Figure 2}

HERG currents exhibit resurgent properties. (A) HERG channel current (I HERG $_{\text {) }}$ expressed in Xenopus oocytes (upper trace) activates (a) and rapidly inactivates (b), causing a suppression of current during the positive voltage step (lower trace), subsequently rebounds in response to repolarization (c), and slowly returns to rest (d) (M. Trudeau and G.A. Robertson, unpublished observations). (B) The corresponding current during an action potential voltage command is demonstrated by HERG channels expressed in HEK 293 cells recorded at physiological temperatures. The current reaches its maximum (dashed vertical line) only after rebounding from inactivation during repolarization during phase 3 of the action potential. Panel $\mathbf{B}$ adapted with permission from Circulation Research (12). corrected QT intervals increased by 33\%, and action potential duration increased by about $75 \%$, as illustrated in Figure 3A. $\mathrm{I}_{\mathrm{Kr}}-$ measured in cardiomyocytes as a cesium current in isolation from the other native currents, which are blocked by $\mathrm{Cs}^{+}$ (18) - was reduced by about one-third. Thus, hypokalemia in vivo produces a stable reduction in native $\mathrm{I}_{\mathrm{Kr}}$ that cannot be attributed to acute effects of reduced $\left[\mathrm{K}^{+}\right]_{\mathrm{o}}$ on inactivation gating. However, a combined effect of the stable reduction of HERG surface expression and acute gating changes could further reduce $\mathrm{I}_{\mathrm{Kr}}$ and increase the risk for hypokalemia-induced TdP.

But does the mechanism of internalization described in the study by Guo et al. (13) account for the changes in $\mathrm{I}_{\mathrm{Kr}}$ magnitude and QT interval prolongation observed in vivo? A dose-response curve for HERG currents in HEK 293 cells after a week in culture at different $\left[\mathrm{K}^{+}\right]_{\mathrm{o}}$ showed that the $\mathrm{EC}_{50}$ is very close to the serum $\left[\mathrm{K}^{+}\right]_{\mathrm{o}}$ (about $2 \mathrm{mM}$ ) achieved in the hypokalemic animal model, leading to the prediction that $\mathrm{I}_{\mathrm{Kr}}$ would indeed be stably reduced in vivo. If there is a quibble with the results, it is that the $\mathrm{EC}_{50}$ for the maintenance of the mature HERG protein in biochemical assays is much lower than that for preserving $\mathrm{I}_{\mathrm{Kr}}$ and normal APD and QT interval. However, these discrepancies may arise because the biochemical assays were conducted before the effects of altered

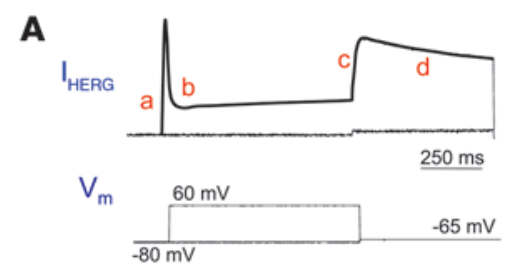

$\mathbf{B}$

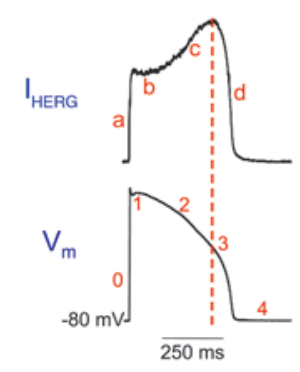


A

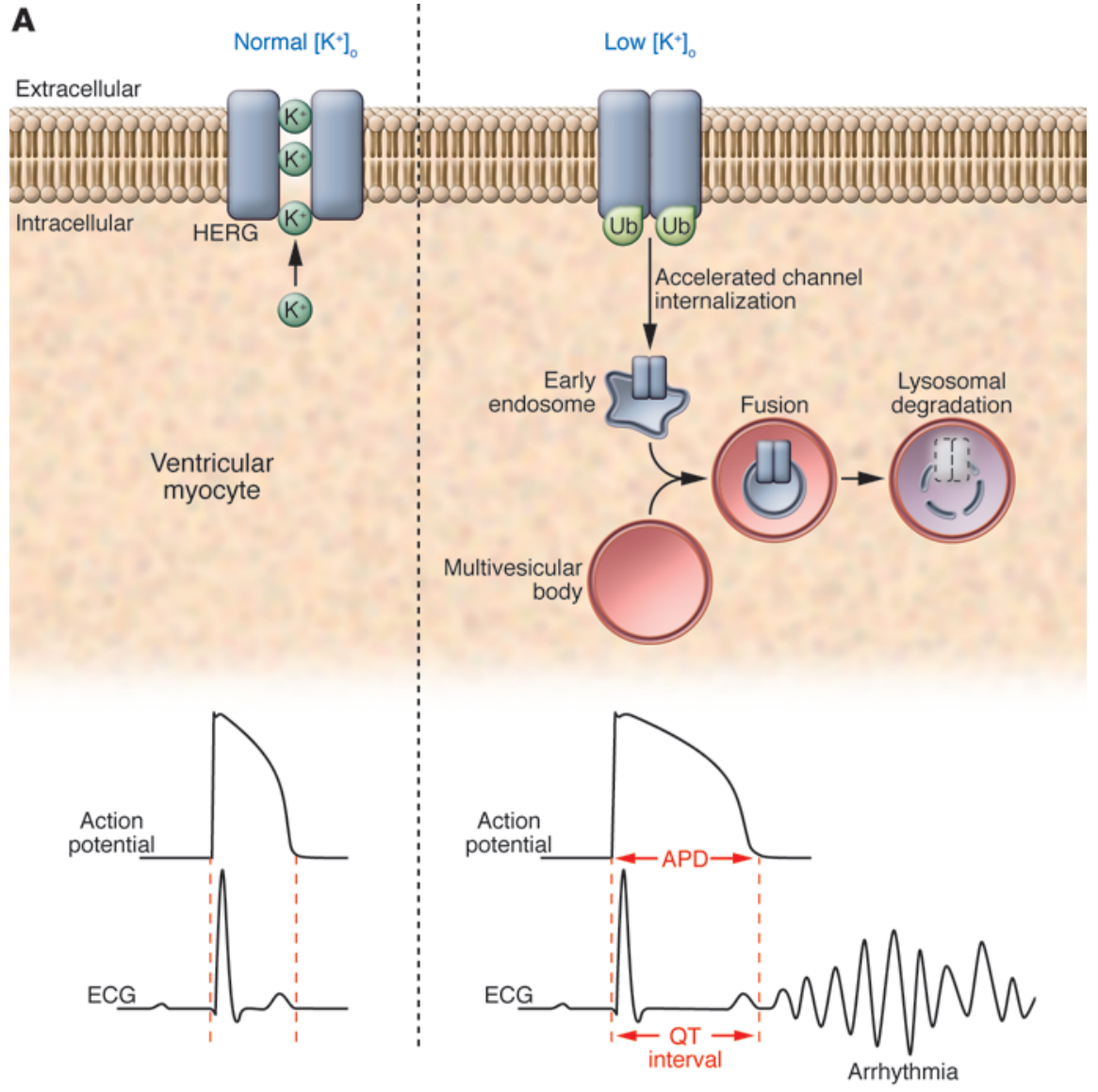

B

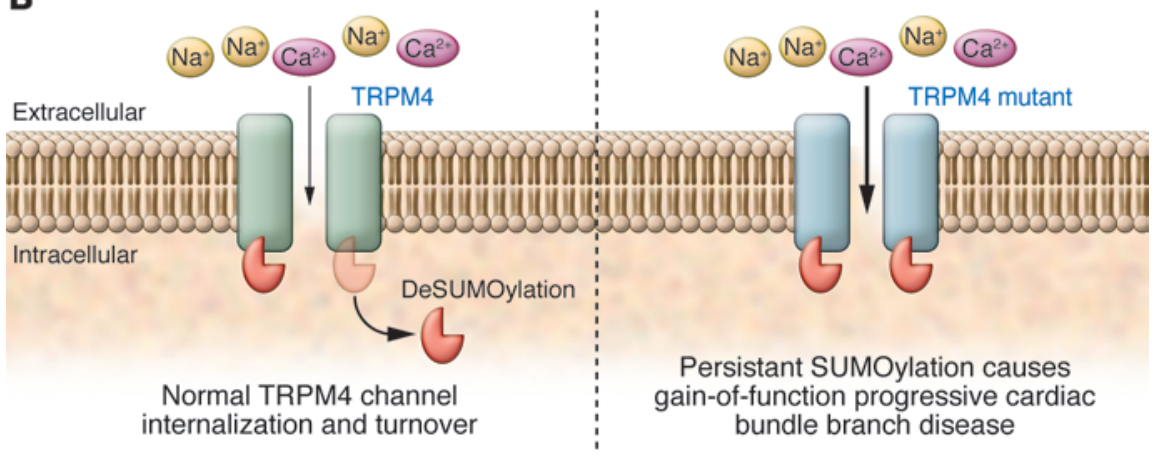

$\left[\mathrm{K}^{+}\right]_{\mathrm{o}}$ reached steady state. Indeed, whereas the $\mathrm{EC}_{50}$ for HERG currents recorded after a week in culture was approximately $2 \mathrm{mM}$ $\left[\mathrm{K}^{+}\right]_{\text {o, }}$, when measured after only 12 hours, it was reduced to sub-mM levels, indicating that more drastic reductions of $\left[\mathrm{K}^{+}\right]_{0}$ were required to achieve the same effect under non-steady-state conditions. The same may be true for the biochemical assays of HERG trafficking conducted before steady state has been reached, in which case the cellular mechanisms for internalization could explain the elevated risk for $\mathrm{TdP}$ even under conditions of relatively mild hypokalemia.

\section{Figure 3}

Two opposing defects in ion channel turnover lead to disease. (A) In their study in this issue of the $\mathrm{JCl}$, Guo et al. (13) show that reduction of external $\left[\mathrm{K}^{+}\right]_{0}$ causes a conformational change in the HERG ion channel that triggers HERG ubiquitination (Ub), internalization, and lysosomal degradation. In the heart, this mechanism causes chronic suppression of $\mathrm{I}_{\mathrm{K} r}$, reduced repolarization following excitation of the heart, and prolongation of the QT interval on the ECG, which can lead to arrhythmia. (B) In the study by Kruse et al. (25), also in this issue, a defect in deSUMOylation is shown to prevent TRPM4 channel internalization and leads to enhanced $\mathrm{Na}^{+}$and $\mathrm{Ca}^{2+}$ influx and progressive cardiac bundle branch disease. 
dose-response curves for maintenance of surface HERG channels give Hill coefficients consistent with the X-ray crystallographic picture of a $\mathrm{K}^{+}$channel selectivity filter fully occupied by 2 ions and 1 in the queue (19). Thus, the idea that evacuation of $\mathrm{K}^{+}$ions and the collapse of the pore proposed for C-type inactivation (9, 20) precede internalization is consistent with these observations. Although closely related to HERG channels, human ethera-go-go (EAG) channels do not inactivate, and their surface density is insensitive to changes in $\left[\mathrm{K}^{+}\right]_{\mathrm{o}}(13)$. The hypothesis that channel inactivation and internalization are causally linked could easily be tested by determining whether the known inactivation mutations confer resistance to HERG internalization in low $\left[\mathrm{K}^{+}\right]_{\mathrm{o}}$. Alternatively, the unstable HERG channel could reflect occupancy of other nonconducting states (21-24) in the plasma membrane prior to internalization.

\section{Defect in deSUMOylation prevents normal TRPM4 channel turnover}

A different mechanism also regulating surface cation channel density in the heart is also described in this issue by Kruse et al. (25). Genetic analysis of a large family with progressive familial heart block type I, an inherited defect in the heart's conduction system, uncovered a mutation leading to misprocessing of transient receptor potential cation channel, subfamily $M$, member 4 (TRPM4). TRPM4 is a $\mathrm{Ca}^{2+}$-activated nonselective cation channel linked to the complex cardiac transient inward current ( $\mathrm{I}_{\mathrm{t} i} ;$ ref. 26). The mutation causes resistance to TRPM4 deSUMOylation, an enzymatic step required for endocytosis and normal channel turnover (Figure 3B). The result is an enhancement of surface channel expression. How this gain of function of TRPM4 causes progressive cardiac bundle branch disease is an important question for future studies, along with defining a clear physiological role for native cardiac channels containing TRPM4.
Now that we have identified the genetic basis for most ion channel subunits, the next big question is how the balance of those ion channels is maintained to carefully control membrane excitability, rhythmic activity, and concentration of ions such as $\mathrm{Ca}^{2+}$, elevation of which can cause cytotoxicity and cell death. The studies by Guo et al. (13) and Kruse et al. (25) share the remarkable finding that modulation of channel densities via defects in surface membrane trafficking can lead to disease and provide a glimpse into what is certain to be a fascinating array of mechanisms involved in this lifesustaining balancing act.

\section{Acknowledgments}

This work was supported by grant HL081780 from the National Heart, Lung, and Blood Institute, NIH.

Address correspondence to: Gail A. Robertson, Department of Physiology, University of Wisconsin - Madison, 601 Science Drive, Madison, Wisconsin 53711, USA. Phone: (608) 265-3339; Fax: (608) 265-7821; E-mail: robertson@physiology.wisc.edu.

1. Dessertenne, F. 1966. Ventricular tachycardia with 2 variable opposing foci. Arch. Mal. Coeur Vaiss. 59:263-272.

2. Roden, D.M., Woosley, R.L., and Primm, R.K. 1986. Incidence and clinical features of the quinidineassociated long QT syndrome: implications for patient care. Am. Heart J. 111:1088-1093.

3. Sanguinetti, M.C., and Jurkiewicz, N.K. 1990. Two components of cardiac delayed rectifier $\mathrm{K}+$ current. Differential sensitivity to block by class III antiarrhythmic agents. J. Gen. Physiol. 96:195-215.

4. Sanguinetti, M.C., and Jurkiewicz, N.K. 1992. Role of external $\mathrm{Ca} 2+$ and $\mathrm{K}+$ in gating of cardiac delayed rectifier K+ currents. Pflugers Arch. 420:180-186.

5. Sanguinetti, M.C., et al. 1995. A mechanistic link between an inherited and an acquired cardiac arrhythmia: HERG encodes the IKr potassium channel. Cell. 81:299-307.

6. Trudeau, M.C., et al. 1995. HERG, a human inward rectifier in the voltage-gated potassium channel family. Science. 269:92-95.

7. Zhou, Z., et al. 1998. Electrophysiological and pharmacological properties of HERG channels in a stably transfected human cell line. Biopbys. J. 74:230-241.

8. Smith, P.L., Baukrowitz, T., and Yellen, G. 1996.
The inward rectification mechanism of the HERG cardiac potassium channel. Nature. 379:833-836.

9. Starkus, J.G., et al. 1997. Ion conduction through C-type inactivated Shaker channels. J. Gen. Physiol. 110:539-550

10. Yang, T., Snyders, D.J., and Roden, D.M. 1997. Rapid inactivation determines the rectification and $[\mathrm{K}+]$ o dependence of the rapid component of the delayed rectifier $\mathrm{K}+$ current in cardiac cells. Circ. Res. 80:782-789.

11. Vandenberg, J.I., et al. 2006. Temperature dependence of human ether-a-go-go-related gene $\mathrm{K}^{+}$ currents. Am. J. Physiol. Cell Physiol. 291:C165-C175.

12. Sale, H., et al. 2008. Physiological properties of hERG 1a/1b heteromeric currents and a hERG $1 \mathrm{~b}$ specific mutation associated with Long-QT syndrome. Circ. Res. 103:e81-e95.

13. Guo, J., et al. 2009. Extracellular $\mathrm{K}^{+}$concentration controls cell surface density of $\mathrm{I}_{\mathrm{Kr}}$ in rabbit hearts and of the HERG channel in human cell lines. J. Clin. Invest. 119:2745-2757.

14. McDonald, T.V., et al. 1997. A minK-HERG complex regulates the cardiac potassium current $\mathrm{I}(\mathrm{Kr})$. Nature. 388:289-292.

15. Abbott, G.W., et al. 1999. MiRP1 forms IKr potassium channels with HERG and is associated with cardiac arrhythmia. Cell. 1999. 97:175-187.

16. Kratz, A., et al. 2004. Case records of the Massachusetts General Hospital. Weekly clinicopathological exercises. Laboratory reference values. N. Engl. J. Med. 351:1548-1563.

17. Garcia, E., et al. 2008. Profound hypokalemia: unusual presentation and management in a 12year-old boy. Pediatr. Emerg. Care. 24:157-160.

18. Zhang, S. 2006. Isolation and characterization of $\mathrm{I}(\mathrm{Kr})$ in cardiac myocytes by Cs+ permeation. Am.J. Physiol. Heart Circ. Physiol. 290:H1038-H1049.

19. Doyle, D.A., et al. 1998. The structure of the potassium channel: molecular basis of $\mathrm{K}+$ conduction and selectivity. Science. 280:69-77.

20. Baukrowitz, T., and Yellen, G. 1996. Use-dependent blockers and exit rate of the last ion from the multiion pore of a $\mathrm{K}+$ channel. Science. 271:653-656.

21. Pardo, L.A., et al. 1992. Extracellular K+ specifically modulates a rat brain $\mathrm{K}+$ channel. Proc. Natl. Acad. Sci. U. S. A. 89:2466-2470.

22. Jager, H., et al. 1998. Regulation of mammalian Shaker-related K+ channels: evidence for non-conducting closed and non-conducting inactivated states. J. Physiol. (Lond.). 506:291-301.

23. Loboda, A., Melishchuk, A., and Armstrong, C. 2001. Dilated and defunct $K$ channels in the absence of $\mathrm{K}^{+}$. Biophys. J. 80:2704-2714.

24. Lopez-Barneo, J., et al. 1993. Effects of external cations and mutations in the pore region on C-type inactivation of Shaker potassium channels. Receptors Channels. 1:61-71.

25. Kruse, M., et al. 2009. Impaired endocytosis of the ion channel TRPM4 is associated with human progressive familial heart block type I. J. Clin. Invest. 119:2737-2744.

26. Guinamard, R., et al. 2006. Calcium-activated nonselective cation channels in mammalian cardiomyocytes. Trends Cardiovasc. Med. 16:245-250. 